\title{
Clinical Presentation of a Patient with Congenital Cutis Laxa and Abnormal Thyroid Hormone Levels
}

\author{
Yan Ma ${ }^{a} \quad$ Jin-yu Zhang ${ }^{a} \quad$ Chen Wang $^{b} \quad$ Wen Cen $^{a} \quad$ Xin Liu $^{a} \quad$ Wen- \\ li Feng ${ }^{a}$ \\ Departments of ${ }^{\mathrm{a}}$ Dermatology and Venerology and ${ }^{\mathrm{b}}$ Pathology, The Second Clinical \\ Medical College, Shanxi Medical University, Taiyuan, China
}

\section{Key Words}

Congenital cutis laxa - Thyroid function - Growth retardation

\begin{abstract}
We describe a case of generalized cutis laxa $(\mathrm{CL})$ in a 7-year-old female child. At 2 months of age, she was found to have a hoarse voice, and at 3 years, she was much smaller than her peers. Her aging face and short stature caught our attention, and the treatment of the patient was accepted by our hospital. She underwent a thorough examination. X-ray of the wrist bone showed a markedly delayed bone age, and thyroid function tests revealed significantly elevated free triiodothyronine 3 and free thyroxine 4 levels, but thyrotropin was within the normal range. Thyroid dysfunction and $\mathrm{CL}$ can be associated with lagged growth and development. Whether her abnormal development was due to thyroid dysfunction or $\mathrm{CL}$ could not be ascertained. $\mathrm{CL}$ is possibly more complex than it has been supposed so far, and is therefore worth to be further studied.

(c) 2014 S. Karger AG, Basel
\end{abstract}

\section{Introduction}

Cutis laxa (CL) is a rare connective tissue disorder caused by defects in the elastic fiber network that can affect multiple tissues, predominantly the skin. Redundant skin is often most noticeable on the neck, hands, and groin, but can also be seen on the face, creating a premature aging appearance [1]. We report a case of a female child with generalized CL, developmental retardation, and abnormal thyroid function. We diagnosed her as having congenital CL with abnormal thyroid hormone levels. 
Congenital CL is a rare, hereditary connective tissue disorder resulting from the abnormality of elastic fibers. The clinical presentation and mode of inheritance show considerable heterogeneity. It can be subdivided into congenital and acquired forms. In congenital CL, three modes of inheritance have been described [2]; more and more gene mutations associated with elastic fibers have been reported [3]. A number of metabolic disorders have also been found to be associated with inherited CL, such as Menkes disease, congenital disorders of glycosylation, and others [4, 5]. In humans, thyroid dysfunction is associated with alterations in skin architecture and homeostasis [6]. In hyperthyroid individuals, the skin often presents with some of the following symptoms: softness, perspiration, heat, itching, generalized pruritus, chronic urticaria, vitiligo, and diffuse skin pigmentation. In addition, the epidermis is usually thinner than normal. In hypothyroid subjects, the skin is dry, cold, and rough. The epidermis is hyperkeratotic, alopecia may develop, and there is diffuse myxedema [7]. A newborn with an autosomal recessive form of congenital CL has been reported in association with congenital hypothyroidism owing to isolated thyrotropin deficiency. This report was the first and the only one to show that CL was related with the thyroid [8]. However, our patient was different from this case, with normal thyrotropin (TSH), higher free triiodothyronine 3 (FT3) and free thyroxine (FT4). A case similar to our patient has not been reported previously. We do not know whether these findings point to a relation between thyroid hormone metabolism and congenital CL.

\section{Case Presentation}

A 7-year-old female patient was admitted to our hospital due to developmental delay, growth retardation, and loose facial skin since birth. At 2 months of age, she was found to have a hoarse voice. At the age of 3 years, her height was significantly lower than that of children of the same age. However, she had no other symptoms, had normal intelligence, and was able to perform normal activities. She underwent surgery to repair an inguinal hernia at the age of 3 years. Her father had a similar skin disease, but his height was normal with about 5.6 feet $(1.7 \mathrm{~m})$. Physical examination revealed that the patient's height was $100 \mathrm{~cm}$, weight $16 \mathrm{~kg}$, and she had loose inelastic skin hanging in folds. Her face appeared like that of an elderly woman (fig. 1a). Loose and sagging skin folded over the neck, arm, and trunk, which gave her a prematurely senile appearance with her skin displaying obvious relaxation and elasticity (fig. 1b-e). The thyroid was not enlarged. Her father also had skin hanging in folds over his abdomen (fig. 2). Further examination after admission revealed a normal electrocardiogram and chest X-ray. Radiological investigation of the hand wrist bone showed a markedly delayed bone age. Laboratory values revealed normal values for complete blood count, erythrocyte sedimentation rate, routine urine analysis, blood biochemistry, hepatic enzymes, and serum electrophoresis. Serologic tests for syphilis (RPR, TPPA) were negative. Skin biopsy specimens taken from the involved area were stained with hematoxylin-eosin and Weigert stains. Histological findings from the skin biopsy revealed a dermal collagen fiber glass-like swelling and perivascular lymphocyte infiltration. Elastic fiber staining showed that the elastic fibers present were fragmented, arranged in disorder, and had a granular appearance (fig. 3a, b). By contrast, the collagen structure, content, and distribution were normal (fig. 3c). Because of the growth delay, the thyroid functions were tested and revealed FT3 $7.71 \mathrm{mmol} / \mathrm{l}$ (normal range: 3.8-6.0), FT4 $24.80 \mathrm{mmol} / \mathrm{l}$ (normal range: 7.914.4), TSH $2.29 \mathrm{mIU} / \mathrm{l}$ (normal range: $0.34-5.0 \mathrm{mIU} / \mathrm{l}$ ), thyroid microsomal antibody $4.16 \%$ (normal range: <15), and thyroglobulin antibody 9.23\% (normal range: <30). Growth hormones and adrenocorticotropic hormones were all within normal ranges, as were skull 
Ma et al.: Clinical Presentation of a Patient with Congenital Cutis Laxa and Abnorma Thyroid Hormone Levels

radiographs. Based on these clinical manifestations and laboratory examinations, the most likely diagnosis in our patient was CL syndrome with abnormal thyroid hormone levels.

\section{Discussion}

CL is characterized by abnormal elastic fibers resulting in loose, redundant, hypoelastic skin. Typically, the skin in CL can easily be pulled away from underlying tissue and only slowly returns to its original position. There are no accurate incidence data available, and there is no known racial or ethnic predilection for CL. CL may be inherited as a dominant, recessive, an X-linked recessive disease or it may be acquired. The heritable forms of CL predominantly begin at birth, but it may be delayed until puberty or later with extracutaneous manifestations including pulmonary emphysema, umbilical and inguinal hernias, and gastrointestinal and vesico-urinary tract diverticuli [9]. As the patient's father had similar clinical manifestations and the generalized laxity of the skin and hernias were associated with CL, we concluded that his daughter had autosomal dominant CL (ADCL). ADCL may present from birth to early adulthood with predominantly skin findings. Systemic manifestations can range from mild to severe, including cardiac and pulmonary complications such as bronchiectasis and emphysema. Many patients live normal life spans, although some of them with ADCL experience serious systemic problems including aortic aneurysms, severe congenital lung disease, and pulmonary artery disease [1]. The clinical presentation and the mode of inheritance show considerable heterogeneity. In the present case, the patient's associated features include developmental delay, and growth retardation without severe systemic complications suggests autosomal dominant inheritance. Severe short stature and dwarfism are common in autosomal recessive CL II, De Barsy syndrome, X-linked recessive CL, gerodermia osteodysplastica, and Costello syndrome [10]. A variety of CL syndromes have also been described, some of them exhibiting mental retardation, but only few reports suggested an association with the abnormal thyroid function. In the present case, FT3 and FT4 were significantly elevated, but TSH was within the normal range. It is unclear if short stature and dwarfism are part of the clinical presentation of CL or instead caused by the abnormal thyroid function. There is no effective drug treatment for anetoderma, but plastic surgery can be considered. Reconstructive surgery provides a dramatic cosmetic improvement with significant psychosocial benefit. Our patient received levothyroxine replacement therapy because of the apparent low thyroid hormone secretion. After 3 months, her parents found no marked changes of the loose skin and height, which led to the discontinuation of the drug. Now, her height is $115 \mathrm{~cm}$, which is significantly lower than that of normal 10-yearolds. Based on this case, CL is likely to be a complex disease accompanied by multiple anomalies, and there may be a dermatological clue to autoimmunity. We suggest that the finding of wrinkled skin or CL in a child with developmental delay should evoke a detailed endocrine and metabolic evaluation.

\section{Acknowledgment}

We thank Dr. William J. Steinbach from the Department of Pediatrics, Duke University Medical Center, Durham, N.C., USA, for his editing. 
Ma et al.: Clinical Presentation of a Patient with Congenital Cutis Laxa and Abnormal Thyroid Hormone Levels

\section{Disclosure Statement}

None of the authors has any conflicts of interest to declare.

\section{References}

1 Berk DR, Bentley DD, Bayliss SJ, Lind A, Urban Z: Cutis laxa: a review. J Am Acad Dermatol 2012;66:842.e1e17.

2 Beighton P: The dominant and recessive forms of cutis laxa. J Med Genet 1972;9:216-221.

-3 Urban Z: The complexity of elastic fiber biogenesis: the paradigm of cutis laxa. J Invest Dermatol 2012;132:E12-E14.

-4 Mohamed M, Kouwenberg D, Gardeitchik T, Kornak U, Wevers RA, Morava E: Metabolic cutis laxa syndromes. J Inherit Metab Dis 2011;34:907-916.

5 Funke S, Gardeitchik T, Kouwenberg D, Mohamed M, Wortmann SB, Korsch E, Adamowicz M, Al-Gazali L, Wevers RA, Horvath A, Lefeber DJ, Morava E: Perinatal and early infantile symptoms in congenital disorders of glycosylation. Am J Med Genet A 2013;161A:578-584.

6 Slominski A, Wortsman J: Neuroendocrinology of the skin. Endocr Rev 2000;21:457-487.

7 Safer JD: Thyroid hormone action on skin. Dermatoendocrinol 2011;3:211-215.

8 Koklu E, Gunes T, Ozturk MA, Akcakus M, Buyukkayhan D, Kurtoglu S: Cutis laxa associated with central hypothyroidism owing to isolated thyrotropin deficiency in a newborn. Pediatr Dermatol 2007;24:525-528.

-9 Gveric T, Baric M, Bulat V, Situm M, Pusic J, Huljev D, Zdilar B, Gveric-Ahmetasevic S, Tomas D: Clinical presentation of a patient with localized acquired cutis laxa of abdomen: a case report. Dermatol Res Pract 2010;2010:402093.

-10 Morava E, Guillard M, Lefeber DJ, Wevers RA: Autosomal recessive cutis laxa syndrome revisited. Eur J Hum Genet 2009;17:1099-1110. 
Ma et al.: Clinical Presentation of a Patient with Congenital Cutis Laxa and Abnormal Thyroid Hormone Levels
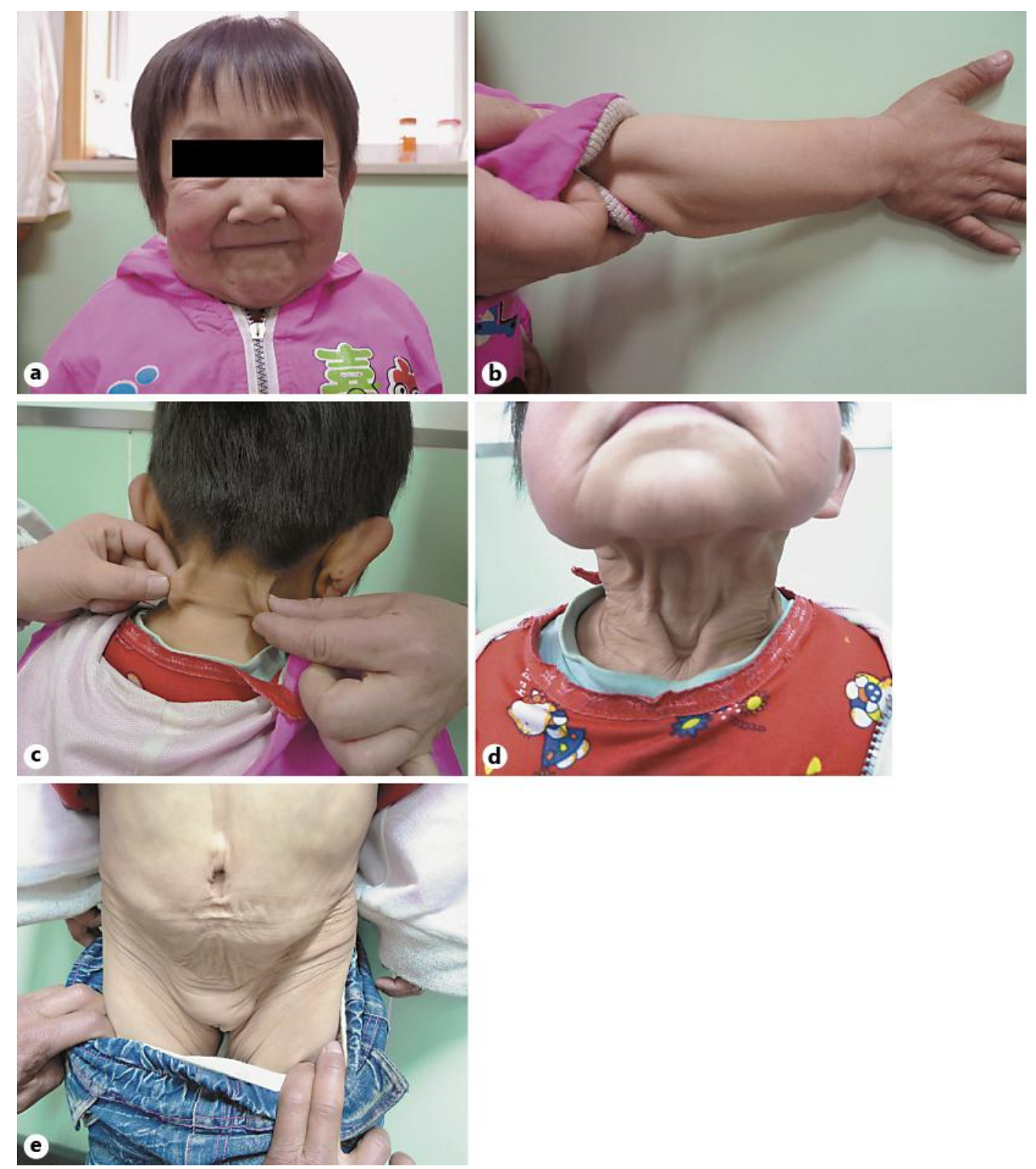

Fig. 1. Skin examination of the 7-year-old female patient. a Old woman's face. b, c Loose skin at the forearm and neck. $\mathbf{d}$ Inelastic skin at the submandibular region after the face-lift. e Sagging of the skin at the groin. 


\begin{tabular}{l|l}
\hline Case Rep Dermatol 2014;6:43-48 \\
\hline DOI: $10.1159 / 000360125$ & $\begin{array}{l}\text { C 2014 S. Karger AG, Basel } \\
\text { www.karger.com/cde }\end{array}$ \\
\hline
\end{tabular}

Ma et al.: Clinical Presentation of a Patient with Congenital Cutis Laxa and Abnormal Thyroid Hormone Levels

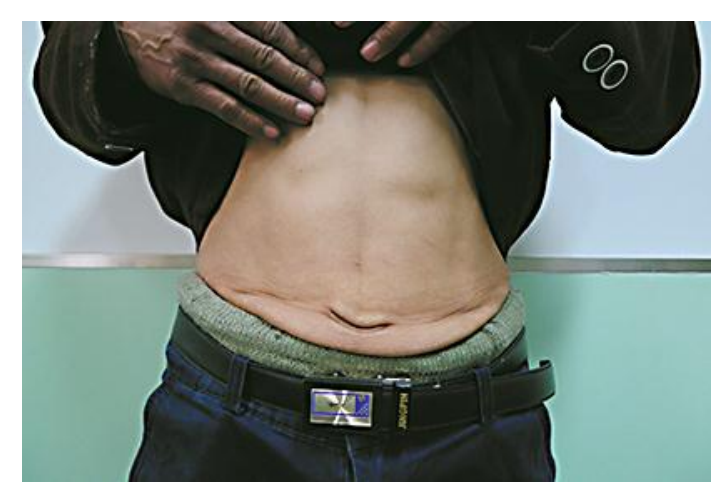

Fig. 2. Skin examination of the patient's father. Inelastic skin at the abdomen.
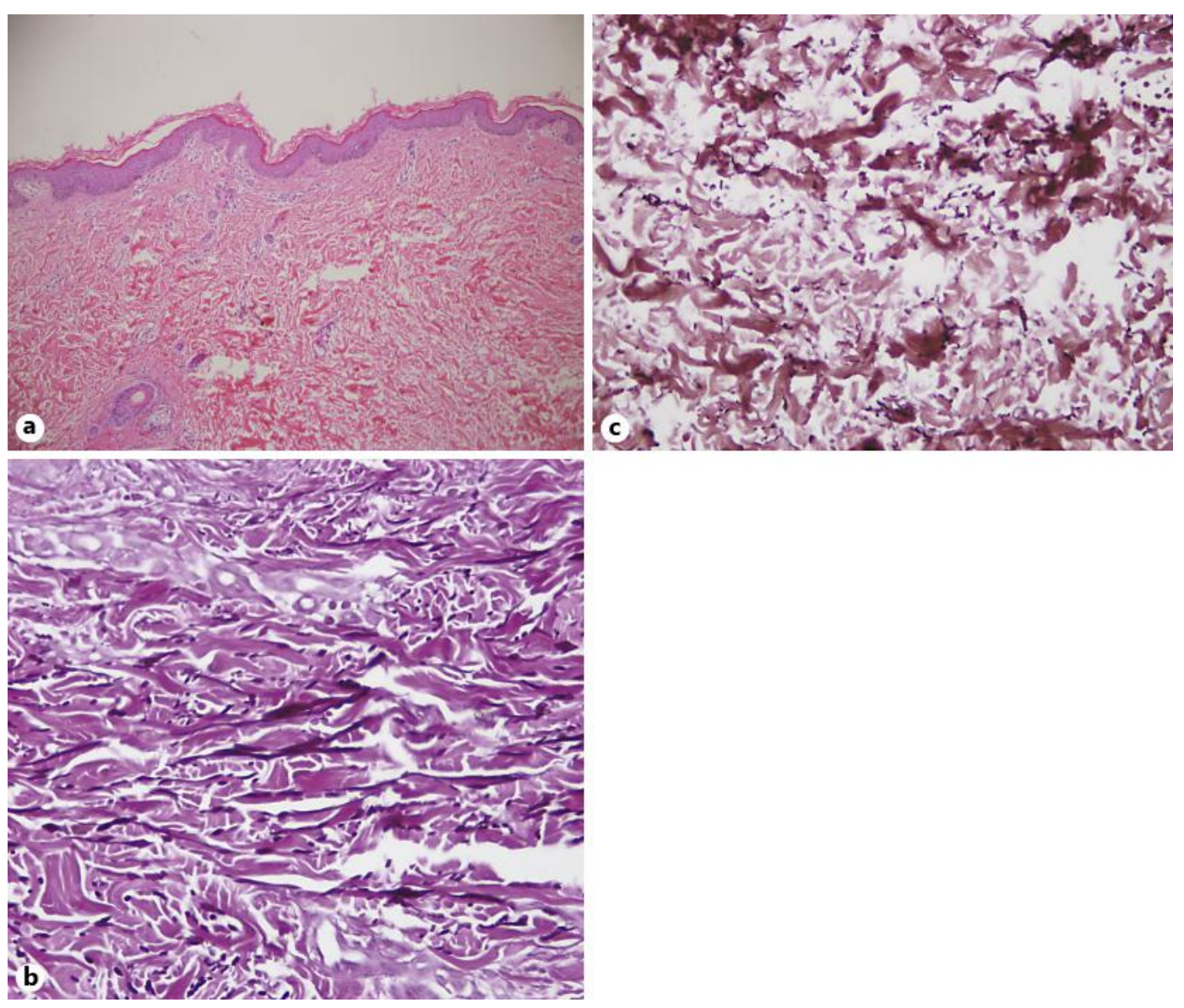

Fig. 3. Histological image of the patient's skin. a Hematoxylin-eosin staining of the skin biopsy specimen shows a hyaline swelling of dermal collagen fibers and small perivascular lymphocytic infiltration. b Weigert elastic fiber staining shows the decrease of elastic fibers that were fragmented and arranged in disorder. c Weigert staining of the skin biopsy specimen from a healthy patient of the patient's age group shows normal elastic fibers. 